prevention of mother-to-child transmission (PMTCT) programme in the teaching hospital.

Design A cross-sectional survey of 236 HIV-positive and $162 \mathrm{HIV}$ negative postpartum women interviewed within 18 months of their expected delivery date in a public-sector health facility providing PMTCT services.

Methods Bi-variant analyses explored fertility intentions, and family planning knowledge and use by HIV status. Multivariate analysis identified socio-demographic and service delivery-related predictors of reporting a desire for additional children and modern family planning use.

Results HIV-positive women were less likely to report wanting additional children than HIV-negative women ( 8 vs. $49 \%$, P < 0.001 ), and although a majority of women reported discussing family planning with a health worker during their last pregnancy (HIV-positive 79\% vs. HIV-negative $69 \%, \mathrm{P}=0.0$ ), modern family planning use remained low in both groups (HIV-positive $43 \%$ vs. HIV-negative $12 \%$, $\mathrm{P}<0.001)$. Condoms were the most commonly used method among HIV positive women (31\%), whereas withdrawal was most frequently reported among HIV-negative women (19\%). In multivariate analysis, HIV-negative women were 16 times more likely to report wanting additional children and nearly $85 \%$ less likely to use modern family planning. Women who reported making two or less antenatal care visits were $77 \%$ less likely to use modern family planning.

Conclusion Our results highlight success in provision of family planning counselling in PMTCT services. As family planning use was low among HIV-positive and negative women, further efforts are needed to improve uptake of modern methods, including dual protection, in the PMTCT settings.

\section{P2.174 VACCINATION AGAINST HPV16/18 INFECTION: IMPACT ON OUALITY OF LIFE}

doi:10.1136/sextrans-2013-051184.0438

\section{J Paavonen. Department of Obstetrics and Gynecology, University Hospital, Helsinki, Finland}

Genital human papillomavirus (HPV) infections and associated precancerous lesions decrease health-related quality of life (HRQoL). Since HPV vaccines protect effectively against these conditions we investigated the impact of HPV vaccination on HRQoL in young women five years after participation in a phase III HPV vaccination trial in comparison to an unvaccinated control cohort. A total of 4808 originally 16 to 17 year-old women had participated in the PATRICIA trial and received either bivalent HPV-16/18 vaccine or hepatitis A-virus (HAV) vaccine in 2004-2005. Unvaccinated adolescent women ( $\mathrm{=}$ 9602), from adjacent birth cohorts, consisted the control cohort. During 2009-2011, all participants received a questionnaire consisting of two generic HRQoL instruments (RAND36 and EQ VAS) and a disease-specific questionnaire (CECA10). We analysed responses of 1143 HPV-16/18 vaccinated-, 980 HAV vaccinated-, and 3753 unvaccinated young women. The unadjusted mean outcome measures of the different HRQoL estimates were similar in the three different responder cohorts. In conclusion, five-years after vaccination the HRQoL of HPV-16/18 vaccinated young women did not differ from that of HAV-vaccinated or unvaccinated controls representing the general population. This was somewhat unexpected, but the study should be repeated after a few years.

\section{P2.175 INCIDENCE AND PREDICTORS OF AIDS RELATED OPPORTUNISTIC ILLNESSES AFTER INITIATION OF HAART: RESULTS FROM A RETROSPECTIVE SINGLE CENTRED COHORT STUDY, AYDER REFERRAL HOSPITAL, MEKELLE UNIVERSITY, ETHIOPIA}

doi:10.1136/sextrans-2013-051184.0439
S Abebe, ${ }^{1} \mathrm{~A}$ Adem, ${ }^{2,3} \mathrm{~N} \mathrm{H}$ Brockmeyer, ${ }^{2} \mathrm{~A}$ Potthoff, ${ }^{2} \mathrm{~J}$ Coenenberg, ${ }^{1} \mathrm{E}$ Bekele, ${ }^{2,4} \mathrm{~A}$ Skaletz-Rorowski, ${ }^{5} Y$ Adama. 'Department of Internal Medicine, College of Health Science, University of Mekelle, Mekelle, Ethiopia; ${ }^{2}$ Clinic for Dermatology, Venerology and Allergology, Ruhr-Universität Bochum, Bochum, Germany; ${ }^{3}$ German STI-Society (DSTIG), Bochum, Germany; ${ }^{4}$ German Competence Network for HIVIAIDS, Bochum, Germany; ${ }^{5}$ Department of Public Health, College of Health Science, University of Mekelle, Mekelle, Ethiopia

Background AIDS related opportunistic illnesses (OIs) have been major causes of morbidity and mortality before and to a lesser extent after the era of highly active antiretroviral therapy (HAART). Studies concerning their magnitude are available from different parts of the world, but are scarce in Ethiopia. The aim of this study was to determine incidence and predictors of AIDS related OIs after initiation of HAART.

Methods A hospital based retrospective cohort study was conducted among HIV patients aged $\geq 14$ years who started HAART in Ayder Referral Hospital, Mekelle/Ethiopia, between January 2009 and May 2012. Simple random sampling was utilised to pick 348 participants, whose data was extracted, cleared and analysed using SPSS version 16 . OIs determinants and correlations were checked using multivariate binary logistic regression model, Odds-ratio and P-value. $\mathrm{P}<0.05$ was considered significant. Kaplan-Meier method was used to estimate OI free survival time after HAART.

Results Incidence of HIV related OIs after HAART was 7.5 cases/100 person-years. Oral candidiasis, disseminated tuberculosis, pneumonia and CNS toxoplasmosis were the leading OIs after HAART. Mean CD4+ count at initiation of HAART was $121 \pm 81 /$ $\mu \mathrm{l}$, Viral-load wasn't documented as it is determined rarely. The median OIs free survival time after HAART was 2 months (1.2-2.9). A bed ridden functional status (OR: 3.8, 1.7-8.4), presence of OIs before HAART (AOR: 2.8, 95\% CI 1-6.9), non-adherence to HAART (AOR: 14.6, CI: 5.8-119), and low haemoglobin level were predictors for occurrence of AIDS related OIs after HAART (AOR: 6.8, 95\% CI: 2-22.4)

Conclusion Incidence of AIDS related OIs after HAART was high. A bed ridden functional status, presence of OIs before HAART, nonadherence for HAART and low haemoglobin level were predictors for AIDS related OIs after HAART initiation. Patients with these risk factors need strict follow up to reduce the morbidity and mortality attributed to OIs.

\section{P2.176 ASSESSING SYNDROMIC MANAGEMENT ALGORITHMS FOR THE DIAGNOSIS OF RECTAL CHLAMYDIA AND GONORRHOEAE AMONG MSM CLINIC ATTENDEES FROM TWO CITIES IN INDIA}

doi:10.1136/sextrans-2013-051184.0440

'P R Mugundu, 'P Narayanan, ${ }^{1} \mathrm{~A}$ Das, ${ }^{2} \mathrm{G}$ Morineau. ${ }^{1} \mathrm{FH} / 360$, New Delhi, India; ${ }^{2}$ FHI360, Bangkok, Thailand

Background Studies assessing algorithms for management of anorectal discharge (ARD) syndrome among men who have sex with men (MSM) are scarce. Performance of ARD management flow charts for the diagnosis of Neisseria gonorrhoeae (NG) and Chlamydia trachomatis (CT) was evaluated.

Methods Between 2008 and 2009, four MSM dedicated clinics, located in two Indian cities, enrolled attendees consecutively and provided follow-up visit during four months. Data collected at each visit included behavioural information, clinical data, and rectal swabs tested for NG and CT using Roche Amplicor. Eight ARD algorithms were assessed. Data were used to construct the best performing flowchart.

Results The 508 participants made a total of 868 clinic visits including 127 instances of rectal NG and/or CT. Among those instances of NG/CT, only one (0.8\%) had ano-rectal complaint(s) and $12(9.5 \%)$ had ano-rectal discharge, observed at clinical 
examination. And on rectal smear microscopy, 31.5\% had pus cells (OR 2.16, $\mathrm{p}<0.001$ ) and $44.1 \%$ had gramme negative diplococci with/without pus cells (OR 1.60, $\mathrm{p}=0.016$ ).

Algorithms using ano-rectal symptom as entry point had poor sensitivity $(0.8 \%)$. The algorithm using receptive anal sex and/or complaint of ano-rectal discharge as the entry point, and subsequently using proctoscopic and/or rectal smear findings had a sensitivity, specificity and positive predictive value of 41.7, 66.3 and 17.5, respectively. By adding risk assessment, the algorithm performance values changed to 81.9, 20.1 and 14.9, respectively.

Conclusion Effectiveness of flow-charts for syndromic management of ARD is constrained by the asymptomatic nature of rectal NG/CT. Algorithms including receptive anal sex at entry point and applying risk assessment, clinical and rectal smear examination may be useful for management of rectal NG/CT in resource limited settings, until cheaper, reliable and feasible laboratory tests are made available.

\section{P2.177 DYSPAREUNIA AMONG WOMEN AGED 40 TO 60 YEARS INFECTED WITH HIV}

doi:10.1136/sextrans-2013-051184.0441

A L R Valadares, L Costa-Paiva, E Amaral, M Souza, A M Pinto-Neto. UNICAMP, Campinas - SP, Brazil

Background Psychosocial aspects of HIV infection and use of medications may be associated with dyspareunia. In middle-aged women there's also problems associated with climacteric symptoms that may influence the presence of dyspareunia, a topic not fully investigated in women with HIV in the ageing process.

Methods Cross sectional study involving 273 women aged 40-60 years. From these 128 women informed having sexual activities with vaginal penetration and were included in the present study. The evaluation instrument was based on the Short Personal Experiences Questionnaire (SPEQ). Sociodemographic, clinical, behavioural, reproductive, HIV issues and partner-related factors were assessed. Dyspareunia was defined as pain during sexual intercourse, graded from 1 to 6 , where a score of 2 or more represented the presence of dyspareunia. A bivariate analysis was performed in which dyspareunia was considered the dependent variable according to the independent variables. The qui-square test was applied with Yates correction or Fisher's exact test.

Results A total of $41.4 \%$ (53) of these women reported dyspareunia. It was associated with low formal education $(p<0.10)$, vaginal dryness $(p<0.001)$, urinary incontinence $(p=0.02)$, muscular pain $(p=0.013)$, partner status HIV + or unknown $(p=0.02)$, and use of statins ( $p>0.05$ and $<0.10$ ). Dyspareunia was less prevalent in women who were in use or previously used biovir $(p<0.05)$ or efavirenz $(p<0.05)$. compared with non-users of these medications.

Conclusions Dyspareunia was common in this cohort of women associated with social, health, partner and medication factors and measures should be adopted to minimise the repercussions of these factors on sexuality.

\section{P2.178 CONDYLOMA ACUMINATA, TREATMENT WITH LIQUID NITROGEN AND TCA}

doi:10.1136/sextrans-2013-051184.0442

1.1 M Ferizi, 'A Gerqari, ${ }^{2} \mathrm{M}$ Ferizi. 'University Clinical Center of Kosovo, Pristina, Kosovo; ${ }^{2}$ University of Kosovo-Medical Faculty, Pristina, Kosovo

Condyloma acuminata is the infection caused by Human Papilloma virus. Condyloma acuminate are soft, skin coloured, fleshy warts, can appear solitary or in a group and can be both small and large. They appear in the vagina, on the cervix, around the external genitalia and rectum, in the urethra and anus. The warts can also appear in nasal, oral and laryngeal form and occasionally occurs in the throat. The disease is highly contagious. The incubation period may take from one to six months Sexually transmitted diseases affect the sexually active population being in the reproductive age group and usually are being transmitted in venereal form. For treatment of genital warts, clinician and patient choose treatment based on morphology and distribution of lesions. During our studies have found that from 226 patients with CA 182 were male and 44 were female. Patients were aged 1-58 years. Treated with liquid nitrogen and own 196 of them were 159 male and 37 female. Treated with TCA were 30 persons, of whom 23 males and 7 females. Treatment with Liquid Nitrogen the majority of patients was extended after 10-15 mal treatment and subjective feeling easily tolerated by patients. Treatment with TCA was the majority of patient successful after first treatment, and the smallest number have had a need to be treated to three times, associated with more severe subjective feeling. Treatment with Liquid Nitrogen is boring, and TCA, although associated with more severe subjective feeling accepted as the most efficient method by patients.

- In total 14 patients smoker treatment was prolonged and resistant to two types of therapy.

- Relapse after treatments were more often in the group of patients treated with Liquid Nitrogen to $3 \%$ of patients.

\section{P2.179 EXPOSURE TO NEISSERIA GONORRHOEAE (GC) DURING THE PERIMENSTRUAL PERIOD MAY INCREASE RISK OF INFECTION IN FEMALE GC CONTACTS}

doi:10.1136/sextrans-2013-051184.0443

S E McLaughlin, J Melendez, K Ghanem, J Zenilman, J M Griffiss. Johns Hopkins School of Medicine, Baltimore, MD, United States

Previous studies have shown that a woman is more likely to be diagnosed with gonococcal (GC) infection during the first five days of her menstrual cycle (active menstruation) than during the rest of her cycle. However, no correlation has been established between risk of infection and the phase of a women's menstrual cycle at the time of exposure. In this study, risk of GC infection was correlated to female menstrual cycle phase according to the woman's stated date of last menstrual period and stated date of GC exposure.

Women ages 16-40 that presented to the Baltimore City Health Department STD Clinic as contacts of known GC positive sexual partners were enrolled. Risk of GC infection was compared to calculated phase of menstrual cycle: perimenstruational period $(-5$ to +5 days since LMP), late follicular (days 6-12), periovulatory (days 13-16), and early luteal (days 17 to -6 day since LMP).

58 female GC contacts were enrolled. Women who presented $>$ 32 days after GC exposure or whose LMP was $>32$ days at presentation where excluded. Of the 29 patients that met inclusion criteria, $11(38 \%)$ were GC negative and $18(62 \%)$ were GC positive (by culture and subsequent NATs). Eighty percent (8/10) of women in the perimenstruational, $38 \%(3 / 8)$ in the late follicular, $80 \%(4 / 5)$ in the periovulatory, and $50 \%(3 / 6)$ in the early luteal phases were GC positive. Fifty-three percent $(7 / 13)$ of $\mathrm{HC}$ users were GC positive.

Increased risk of GC infection during the perimenstrual period may be, in part, due to lower lactoferrin concentration, lower level of iron sequestration, and higher level of free iron in the female genital tract. Increased risk of GC infection surrounding ovulation may be due to higher lutropin receptor (known GC ligand) expression in the female GU tract at ovulation.

\section{P2.180 TMR5 (ZEDUPEXTM) AS A MANAGEMENT THERAPY FOR HERPES INFECTIONS: RESULTS OF PRECLINICAL EVALUATIONS}

doi:10.1136/sextrans-2013-051184.0444

${ }^{1} \mathbf{F}$ M Tolo, 'L Keter, ${ }^{2} \mathrm{G}$ M Mungai. 'Kenya Medical Research Institute, Nairobi, Kenya; ${ }^{2}$ The East African Herbarium, Nairobi, Kenya 